\title{
Lumacaftor/ivacaftor-associated health stabilisation in adults with severe cystic fibrosis
}

\author{
Susannah J. King ${ }^{1,2,3}$, Dominic Keating $\mathbb{1}^{2,4}$, Elyssa Williams ${ }^{2}$, Eldho Paul ${ }^{5}$, \\ Brigitte M. Borg ${ }^{6}$, Felicity Finlayson (10) ${ }^{2}$, Brenda M. Button ${ }^{2,4,7}$, \\ John W. Wilson 2,4 and Tom Kotsimbos ${ }^{2,4}$
}

Affiliations: ${ }^{1}$ Nutrition Dept, Alfred Hospital, Melbourne, Australia. ${ }^{2}$ Cystic Fibrosis Service, Dept of Respiratory Medicine, Alfred Hospital, Melbourne, Australia. ${ }^{3}$ Dept of Dietetics, Nutrition and Sport, LaTrobe University, Bundoora, Australia. ${ }^{4}$ Dept of Medicine, Monash University, Melbourne, Australia. ${ }^{5}$ School of Public Health and Preventive Medicine, Monash University, Melbourne, Australia. ${ }^{6}$ Physiology Service, Dept of Respiratory Medicine, Alfred Hospital, Melbourne, Australia. ${ }^{7}$ Physiotherapy Dept, Alfred Hospital, Melbourne, Australia.

Correspondence: Susannah J. King, Nutrition Dept, Alfred Hospital, Commercial Road, Melbourne, Victoria 3004, Australia. E-mail: s.kingdalfred.org.au

\section{ABSTRACT}

Introduction: Lumacaftor/ivacaftor (LUM/IVA) has been shown to improve clinical outcomes in cystic fibrosis (CF) patients homozygous for Phe508del with forced expiratory volume in $1 \mathrm{~s}\left(\mathrm{FEV}_{1}\right) \%$ pred $>40 \%$. We assessed the clinical utility of LUM/IVA in all eligible adult CF patients with $\mathrm{FEV}_{1} \%$ pred $<40 \%$ treated for at least 1 year under a single-centre managed access programme.

Methods: Following clinical optimisation, eligible patients $(n=40)$ with $\mathrm{FEV}_{1} \%$ pred $<40 \%$ were commenced on LUM/IVA and monitored for tolerance and clinical outcomes, including health service utilisation, pulmonary function, weight and body composition. 24 patients reached 1 year of treatment by the time of evaluation. Six patients discontinued due to adverse events (five for increased airways reactivity) and three underwent lung transplantation.

Results: In comparison with the year prior to LUM/IVA commencement, significant reductions (median per year) were observed in the treatment year in the number of pulmonary exacerbations requiring hospitalisation (from 3 to $1.5 ; \mathrm{p}=0.0002$ ), hospitalisation days (from 27 to $17 ; \mathrm{p}=0.0002$ ) and intravenous antibiotic (IVAB) usage days (from 45 to $27 ; \mathrm{p}=0.0007$ ). Mean \pm SD change in $\mathrm{FEV}_{1} \%$ pred was $-2.10 \pm 1.18 \%$ per year in the year prior, with the decline reversed in the year following $(+1.45 \pm 1.13 \%$ per year; $p=0.035)$, although there was significant heterogeneity in individual responses. Mean \pm sD weight gain at 1 year was $2.5 \pm 4.1 \mathrm{~kg}$ ( $\mathrm{p}=0.0007$ ), comprising mainly fat mass (mean $2.2 \mathrm{~kg}$ ). The proportion of patients severely underweight (body mass index $<18.5 \mathrm{~kg} \cdot \mathrm{m}^{-2}$ ) decreased from $33 \%$ at baseline to $13 \%$ at 1 year $(\mathrm{p}=0.003$ ).

Conclusion: This real-world evaluation study demonstrated benefits over several clinical domains (infective exacerbations requiring hospitalisation, IVABs, pulmonary function decline and nutritional parameters) in CF patients with severe lung disease.

@ERSpublications

In adults with severe cystic fibrosis lung disease, 1 year of treatment with lumacaftor/ivacaftor was associated with reduced infective exacerbations, days of intravenous antibiotics and rate of pulmonary function decline, and improved nutritional status https://bit.ly/2I07suR

Cite this article as: King SJ, Keating D, Williams E, et al. Lumacaftor/ivacaftor-associated health stabilisation in adults with severe cystic fibrosis. ERJ Open Res 2021; 7: 00203-2020 [https://doi.org/ $10.1183 / 23120541.00203-2020]$.

Copyright $\odot$ ERS 2021. This article is open access and distributed under the terms of the Creative Commons Attribution Non-Commercial Licence 4.0 


\section{Introduction}

Lumacaftor/ivacaftor (LUM/IVA) is a combination cystic fibrosis transmembrane conductance regulator (CFTR) modulator therapy proven to be useful in individuals with CF with two copies of the Phe508del mutation and was the first such agent available for this CF genotype [1]. In clinical trials involving patients with mild or moderate lung disease (forced expiratory volume in $1 \mathrm{~s}\left(\mathrm{FEV}_{1}\right) \%$ pred $40-90 \%$ ), LUM/IVA was associated with modestly improved pulmonary function and weight, and reduced pulmonary exacerbation [1-4].

However, LUM/IVA-eligible patients with severe lung disease $\left(\mathrm{FEV}_{1} \%\right.$ pred $\left.<40 \%\right)$ were excluded from these clinical trials. Thus, our understanding of its impact in this group of patients is limited. Murer et al. [5] studied $20 \mathrm{CF}$ adults and reported modest increases in $\mathrm{FEV}_{1} \%$ pred of $2.5 \%$ from a mean baseline $\mathrm{FEV}_{1} \%$ pred of $32 \%$ in the 10 patients that were able to tolerate the medication and remained on it for 6 months, as well as reduced pulmonary exacerbation rate and $\sim 0.9 \mathrm{~kg} \cdot \mathrm{m}^{-2}$ increase in body mass index (BMI). Another observational study in 35 patients with severe lung disease $\left(\mathrm{FEV}_{1} \%\right.$ pred $\left.<40 \%\right)$ completing 24 weeks on LUM/IVA reported a reduction in hospitalisations and duration of intravenous antibiotic (IVAB) usage compared with the 24 weeks prior to commencement [6]. However, the use of these medications at the more severe end of the CF spectrum still needs to proceed with caution. This is pertinent given the not insignificant rates of adverse events and need for discontinuation, with all that this entails both clinically and psychologically regarding unmet expectations [7].

LUM/IVA was not available in Australia until late 2018 outside a clinical trial setting, except via a compassionate use managed access programme for individuals aged $>12$ years, with $\mathrm{FEV}_{1} \%$ pred $<40 \%$, rapidly declining pulmonary function or lung transplant listing. The Alfred Adult CF Service (Melbourne, Australia) is one of the largest adult CF centres in the southern hemisphere, caring for over 340 adults with CF. Severe lung disease $\left(\mathrm{FEV}_{1} \%\right.$ pred $\left.<40 \%\right)$ is an indication for assessment and consideration of lung transplant [8]. In this setting, following care optimisation, LUM/IVA was able to be offered to patients with severe CF lung disease with two copies of the Phe508del mutation via the compassionate use programme, undertaken in conjunction with referral to the lung transplant team and to help manage potential concerns of adverse drug reactions precipitating further respiratory decline. Importantly, low baseline pulmonary function and frequent pulmonary exacerbations requiring hospitalisation are major signals for increased mortality rates in CF and therefore for lung transplant consideration [8]. Hence, the real-world implementation of LUM/IVA treatment in severe CF lung disease needed to proceed within this framework and with caution $[2,5]$.

Pulmonary function and nutritional status are strongly correlated in CF [9]. While it has long been appreciated that higher $\mathrm{FEV}_{1} \%$ pred is associated with higher BMI and with indices of fat-free mass [9-13], the significance of altered body composition is increasingly being recognised [11, 13-16]. Fat-free mass depletion is associated with more severe CF lung disease, increased pulmonary exacerbation rate and higher systemic inflammatory cytokines such as interleukin-6 [17-19]. Changes in weight, fat-free mass and fat mass are not always aligned, underpinning the importance of body composition assessment in both routine clinical care and evaluating novel interventions such as CFTR modulator therapies [19-21]. However, to date there are no data reporting the effect of LUM/IVA on body composition.

Systematically evaluating the effects of CFTR modulator therapy across multiple clinical domains in a single-centre cohort of CF patients with severe lung disease therefore offers a unique opportunity to better understand the potential benefits of these new therapies. In this clinical evaluation study, we aimed to examine the clinical effects (hospitalisation for antibiotic treatment of pulmonary exacerbations, and changes in pulmonary function, weight and body composition) of 1 year of treatment with compassionate access LUM/IVA in adult CF patients with severe lung disease ( $\mathrm{FEV}_{1} \%$ pred $<40 \%$ at baseline).

\section{Methods}

This clinical evaluation study reports on data from all adult patients ( $\geqslant 18$ years) who received LUM/IVA under the care of the Alfred Adult CF Service for at least 1 year, from January 2016 to July 2018, under the managed access programme. This cut-off was chosen because LUM/IVA was approved for government subsidy in August 2018. All patients who became eligible during this period were identified and approached, and all accepted treatment. The study was approved by The Alfred Health Research and Ethics Committee (approval 464/18).

Following clinical evaluation of suitability to commence treatment (including homozygosity for the Phe508del mutation, $\mathrm{FEV}_{1} \%$ pred $<40 \%$ and an absence of key contraindications, e.g. severe liver disease (Child-Pugh C), current severe infective exacerbation or bronchoconstriction), eligible CF patients were hospitalised and treatment regimens optimised prior to the first dose of LUM/IVA. This included pre-emptive management of airway sepsis and airways reactivity (if present) utilising parenteral antibiotic 
treatment as indicated and the use of inhaled ipratropium and inhaled/oral steroids as required, respectively. As is the usual practice at every admission, fluid and nutritional intake, and physiotherapy using airway clearance and exercise, were optimised to ensure baseline functional levels as much as possible. LUM/IVA (supplied by Vertex Pharmaceuticals, Boston, MA, USA) was initiated at half dose and increased to full dose (two 200/125 mg tablets orally every $12 \mathrm{~h}$ ) after a few days to 1 week as tolerated. Patients were educated on taking LUM/IVA every $12 \mathrm{~h}$ with dietary fat. Patients were routinely monitored during inpatient stays and outpatient clinics for tolerance to LUM/IVA (including full clinical assessments, spirometry testing, routine biochemistry (including tests of liver function) and nutritional status). Identification and management of pulmonary exacerbations followed usual standard care practices. Any decision to cease treatment due to adverse events or tolerance issues was made by the treating team. LUM/ IVA was ceased if patients underwent lung transplantation. Where LUM/IVA treatment was ceased prior to 1 year of treatment, specific reasons for this decision were recorded.

For this clinical evaluation study, data were analysed for all adults who reached 1 year of LUM/IVA treatment. Demographic and clinical data at commencement of LUM/IVA (age, sex, $\mathrm{FEV}_{1} \%$ pred, use of supplementary oxygen, diagnosis of CF-related liver disease or CF-related diabetes mellitus and whether listed for lung transplant prior to commencement of LUM/IVA) were collected. Pulmonary function ( post-bronchodilator spirometric data for $\mathrm{FEV}_{1}, \mathrm{FEV}_{1} \%$ pred, forced vital capacity (FVC), FVC \% pred, forced expiratory flow at $25-75 \%$ of $\mathrm{FVC}\left(\mathrm{FEF}_{25-75 \%}\right)$ and $\mathrm{FEF}_{25-75 \%} \%$ pred) and weight measurements for the year prior to commencement of LUM/IVA and the year after commencement were extracted from clinical records (using data from all pulmonary function tests undertaken). At our centre, the predominant indication for IVABs is pulmonary exacerbation, and these courses may be completed in hospital or undertaken partly in hospital and partly at home. Continuation of IVAB courses at home is based on factors including clinical stability, patient ability to manage line care, IVAB delivery and other aspects of the CF care at home, social and geographic factors including patient preference, and for patients living outside the Melbourne region, availability of a local provider of hospital-in-the-home nursing services and distance from their home to the CF centre. Access to home IVAB therapy is available across all $\mathrm{FEV}_{1}$ ranges. All patients are trained in IVAB delivery and must complete a competency checklist prior to transfer to home care. Clinical criteria and practices for home IVAB therapy were consistent across the period of this study. The number of pulmonary exacerbations requiring hospitalisation, days in hospital and days of IVAB usage during the year prior to commencement of LUM/IVA and during the year after commencement were recorded.

Body composition measurement was undertaken by trained operators using multifrequency bioelectrical impedance analysis (mBCA 515/514; SECA, Hamburg, Germany), a method with good reproducibility and validity against reference methods [22]. Measurements were taken prior to commencement of LUM/IVA, and after 1 and 6 months and 1 year of treatment. Body composition variables collected included weight, fat-free mass, fat mass, total body water (TBW), extracellular water (ECW) and intracellular water, ECW as a percentage of TBW, and phase angle. BMI, fat-free mass index (FFMI) and fat mass index (FMI) were calculated by dividing weight, fat-free mass and fat mass $(\mathrm{kg})$ by the square of height $\left(\mathrm{m}^{2}\right)$. Fat-free mass depletion was defined as FFMI $<15 \mathrm{~kg} \cdot \mathrm{m}^{-2}$ for females and $<17 \mathrm{~kg} \cdot \mathrm{m}^{-2}$ for males [23]. "Hidden" fat-free mass depletion was defined as fat-free mass depletion in patients with $\mathrm{BMI} \geqslant 18.5 \mathrm{~kg} \cdot \mathrm{m}^{-2}$ [11].

\section{Statistical analysis}

Statistical analyses were performed using Stata version 15 (StataCorp, College Station, TX, USA) or SAS version 9.4 (SAS Institute, Cary, NC, USA). Size calculation was not undertaken as data from all available patients were included. Continuous data were assessed for normality and reported as mean with standard deviation or median (interquartile range (IQR) or range) depending on the underlying distribution. Differences in health service utilisation variables (number of exacerbations requiring hospitalisation, days in hospital and days of IVAB treatment) between the year prior to commencing LUM/IVA and the year following commencement were compared using Wilcoxon signed-rank tests. For pulmonary function parameters and weight for the year prior to and the year following commencement of LUM/IVA, slope estimates with standard errors were derived for each patient using all measurements for each time period via linear regression modelling to determine annual rates of change pre- and post-LUM/IVA. Comparisons were made using weighted linear regression analyses with weights derived by the inverse variance method, in order to account for the number of measurements per person in each time period. Changes in body composition variables in the year following LUM/IVA were assessed. To account for repeat measures, these data were analysed using linear mixed effects regression modelling, fitting main effect for time with patients treated as random effects. Post hoc comparisons of time-points were performed with Bonferroni correction for multiple comparisons. McNemar's test of paired proportions was used to compare BMI distribution at baseline and 1-year follow-up. Correlations between variables were assessed using Pearson (r) or Spearman ( $\rho$ ) rank correlation wherever appropriate. A formal power calculation was not undertaken as data from all 
available patients were included. However, a retrospective sample size calculation was performed for fat-free mass change. Based on a clinically important mean change of 5\% and the standard deviation of change in fat-free mass at 1 year at power of $80 \%$ and $\alpha$ of 0.05 , a sample size of $n=7$ would have been sufficient to detect this effect size, suggesting the lack of significant change in mean fat-free mass is not due to a type II error. A two-sided $\mathrm{p}$-value $<0.05$ indicated statistical significance.

\section{Results}

Between January 2016 and July 2018, 40 adults with severe CF lung disease commenced LUM/IVA treatment under the managed access programme. All were homozygous for the Phe508del mutation. Of these, 24 had reached 1 year of treatment by July 2018. Six patients ceased due to not tolerating LUM/IVA (airway reactivity $(n=5)$ or significant deterioration in liver function tests $(n=1))$ and three patients underwent lung transplantation, while seven patients had not yet reached 1 year of treatment. These 16 patients had similar mean \pm SD age $(31.8 \pm 9.2$ years; $\mathrm{p}=0.78)$ and $\mathrm{FEV}_{1} \%$ pred $(34.3 \pm 7.9 \% ; \mathrm{p}=0.87)$ to the 24 adults who reached 1 year of treatment, on whom this report is based. Data acquisition was complete other than for three out of 96 missing body composition data points (unanalysable data for one patient at baseline and 1 month, and one patient missed the 6-month measurement). Table 1 shows demographic and clinical characteristics at commencement of LUM/IVA. While no patient was on the waitlist for lung transplant at commencement, two patients were listed during the first year of LUM/IVA treatment (but not yet transplanted by 1 year). No patients were diagnosed with allergic bronchopulmonary aspergillosis requiring antifungal treatment and no substrates of cytochrome P450 3A4 were required. Of the six patients who did not tolerate LUM/IVA, their baseline clinical characteristics were similar to the cohort of 24 patients who completed 1 year of treatment (mean \pm SD $\mathrm{FEV}_{1} \%$ pred 31.7 $\pm 7.0 \%$; $\mathrm{p}=0.38$ and BMI $22.1 \pm 1.9 \mathrm{~kg} \cdot \mathrm{m}^{-2} ; \mathrm{p}=0.14$; with $50 \%$ having CF-related diabetes and $33 \%$ having CF-related liver disease and none on supplemental oxygen).

Table 2 shows hospitalisation and IVAB usage data for the year prior to and the year following commencement on LUM/IVA. The median numbers of pulmonary exacerbations requiring hospitalisation, total days in hospital and days of IVAB usage all decreased significantly (table 2 and figure 1). 11 patients had $>14$ days fewer hospital days in the first year on LUM/IVA compared with the previous year. For IVAB usage, 21 patients had fewer days on IVAB in the first year on LUM/IVA (range 1-247 fewer days) (figure 1). There were no significant sex differences in hospitalisation or IVAB days.

Significant differences in the rates of change in $\mathrm{FEV}_{1} \%$ pred and weight were seen in the year following LUM/IVA treatment compared with the year prior to commencement, with reversal of the patterns of decline for both $\mathrm{FEV}_{1} \%$ pred and weight (table 2). A waterfall plot of the linear regression line slopes for

\section{TABLE 1 Demographics and cystic fibrosis (CF) therapies in 24 adults with CF at baseline (prior to commencement of lumacaftor/ivacaftor)}

\begin{tabular}{|c|c|}
\hline Male & 54.2 \\
\hline Age years & $32.6 \pm 8.6$ \\
\hline Height $\mathrm{cm}$ & $169.0 \pm 10.4$ \\
\hline $\mathrm{BMI} \mathbf{k g} \cdot \mathrm{m}^{-2}$ & $20.3 \pm 2.7$ \\
\hline $\mathrm{FEV}_{1} \mathrm{~L}$ & $1.30 \pm 0.41$ \\
\hline $\mathrm{FEV}_{1} \%$ pred & $34.7 \pm 7.4$ \\
\hline FVC L & $2.65 \pm 0.81$ \\
\hline FVC \% pred & $57.8 \pm 10.0$ \\
\hline $\mathrm{FEF}_{25-75 \%} \mathrm{~L}$ & $0.51 \pm 0.19$ \\
\hline FEF $_{25-75 \%} \%$ pred & $13.3 \pm 4.2$ \\
\hline Diagnosis of CF-related diabetes mellitus & 33.3 \\
\hline Diagnosis of CF-related liver disease & 33.3 \\
\hline Use of supplementary oxygen therapy & 0.0 \\
\hline Azithromycin & 87.5 \\
\hline Recombinant human DNase & 58.3 \\
\hline Hypertonic saline & 58.3 \\
\hline Inhaled antibiotics & 41.4 \\
\hline Oral antibiotics & 33.3 \\
\hline Inhaled corticosteroids & 87.5 \\
\hline Oral corticosteroids & 4.2 \\
\hline Long-acting $\boldsymbol{\beta}_{2}$-agonists & 91.7 \\
\hline Long-acting muscarinic antagonists & 4.2 \\
\hline
\end{tabular}

Data are presented as $\%$ or mean \pm SD. BMI: body mass index; $\mathrm{FEV}_{1}$ : forced expiratory volume in $1 \mathrm{~s}$; FVC: forced vital capacity; $\mathrm{FEF}_{25-75 \%}$ : forced expiratory flow at $25-75 \%$ of FVC. 


\begin{tabular}{|c|c|c|c|}
\hline & $\begin{array}{l}\text { Year prior to } \\
\text { commencement }\end{array}$ & $\begin{array}{l}\text { Year following } \\
\text { commencement }\end{array}$ & p-value \\
\hline \multicolumn{4}{|l|}{ Health service utilisation data } \\
\hline $\begin{array}{l}\text { Pulmonary exacerbations requiring } \\
\text { hospitalisation } \mathrm{n}\end{array}$ & $3(2-4)$ & $1.5(1-2)$ & 0.0002 \\
\hline Hospitalisation (on ward) days & 27 (18-58) (range 10-103) & 17 (10-25) (range 1-70) & 0.0002 \\
\hline IVAB usage days & 45 (21-75) (range 10-280) & 27 (11-52) (range 1-167) & 0.0007 \\
\hline \multicolumn{4}{|l|}{$\begin{array}{l}\text { Annual rate of change in pulmonary } \\
\text { function and weight }\end{array}$} \\
\hline $\mathrm{FEV}_{1} \mathrm{~L}$ & $-0.084 \pm 0.046$ & $0.027 \pm 0.041$ & 0.077 \\
\hline FVC L & $-0.04 \pm 0.10$ & $0.00 \pm 0.06$ & 0.75 \\
\hline $\mathrm{FEF}_{25-75 \%} \mathrm{~L}$ & $-0.07 \pm 0.02$ & $0.02 \pm 0.02$ & 0.007 \\
\hline FEV $_{1} \%$ pred & $-2.10 \pm 1.18$ & $1.45 \pm 1.13$ & 0.035 \\
\hline FVC \% pred & $-0.69 \pm 2.11$ & $1.27 \pm 1.51$ & 0.46 \\
\hline $\mathrm{FEF}_{25-75 \%} \%$ pred & $0.07 \pm 0.20$ & $0.07 \pm 0.05$ & 0.97 \\
\hline Weight kg & $-0.62 \pm 0.89$ & $2.60 \pm 0.88$ & 0.013 \\
\hline
\end{tabular}

Health service utilisation data are presented as median (interquartile range); pulmonary function and weight data show mean change per year (slope \pm SE) using linear regression modelling of all clinical measurements in each year-long period. IVAB: intravenous antibiotic; $F_{E V}$ : forced expiratory volume in $1 \mathrm{~s}$; FVC: forced vital capacity; $\mathrm{FEF}_{25-75 \%}$ : forced expiratory flow at $25-75 \%$ of FVC. p-value for difference between year prior and year following commencement (Wilcoxon signed-rank test for health service utilisation data; weighted linear regression analyses for pulmonary function and weight data).

change in $\mathrm{FEV}_{1} \%$ pred in the year following commencement of LUM/IVA reveals the individual patient response heterogeneity and three out of 15 positive responders having a response of $\geqslant 10 \%$ (figure 1 ). Sensitivity analyses were performed excluding the outlier for each of $\mathrm{FEV}_{1} \%$ pred change, change in IVAB days and change in hospital days, and the significance of each result was retained, showing that the overall results were not driven by single patient responses.

In keeping with the severity of lung disease in this cohort, a third of patients were underweight (BMI $<18.5 \mathrm{~kg} \cdot \mathrm{m}^{-2}$ ) at commencement of LUM/IVA treatment. However, half of the patients had fat-free mass depletion at baseline, in five $(42 \%)$ of whom the fat-free mass depletion was hidden (i.e. low FFMI with $\mathrm{BMI} \geqslant 18.5 \mathrm{~kg} \cdot \mathrm{m}^{-2}$ ).

No changes were observed in weight or body composition in the first month on LUM/IVA treatment. Increase in mean weight was observed during the first 6 months, plateauing by 1 year (table 3 and figure 2). At 1 year, mean \pm SD weight gain was $2.5 \pm 4.1 \mathrm{~kg}(\mathrm{p}=0.0007)$, or a mean percentage increase from baseline of $4.9 \pm 7.3 \%(p=0.0002)$. Half of the patients gained $>5 \%$ of baseline weight, but only one lost $>5 \%$ of baseline weight. Similarly, BMI increased significantly at 1 year $\left(0.90 \pm 1.40 \mathrm{~kg} \cdot \mathrm{m}^{-2} ; \mathrm{p}=0.001\right)$ (table 3). Fat mass increased significantly in the first 6 months, plateauing by 1 year (mean total gain $2.2 \mathrm{~kg}$ ) (table 3 and figure 2). In contrast, there was no overall change in fat-free mass or FFMI, indicating preservation of fat-free mass stores over the first year of treatment (table 3 and figure 2); however, $20 \%$ of patients gained $>5 \%$ of baseline fat-free mass in the year of treatment.

The proportion of patients severely underweight (BMI $<18.5 \mathrm{~kg} \cdot \mathrm{m}^{-2}$ ) decreased from $33 \%$ at baseline to $13 \%$ at 1 year of treatment ( $\mathrm{p}=0.003$ using McNemar's test). In contrast, there was no reduction in the proportion with fat-free mass depletion between baseline and 1 year ( $50 \%$ at both time-points). One patient had a BMI $>25 \mathrm{~kg} \cdot \mathrm{m}^{-2}$ throughout the observational period, but no new cases of overweight emerged. Baseline BMI was inversely correlated with change in weight $(r=-0.54, p=0.006)$, \% change in weight $(\mathrm{r}=-0.56, \mathrm{p}=0.005)$ and change in fat-free mass $(\mathrm{r}=-0.57, \mathrm{p}=0.005)$, but not change in fat mass $(\mathrm{r}=-0.32, \mathrm{p}=0.14)$, indicating that those with the poorest baseline nutritional status experienced the greatest gains in weight and fat-free mass. No sex differences in patterns of change in body composition were observed. Neither age nor pulmonary function correlated with changes in body composition (data not shown). However, change in fat-free mass by 1 year correlated with greater reduction in the number of both hospitalisation days and IVAB days in the year following commencement of LUM/IVA compared with the year prior $(\rho=-0.50, p=0.001$ and $\rho=-0.43, p=0.04$, respectively). Change in weight in the year following commencement was correlated with greater reduction in hospitalisation days $(\rho=-0.48, p=0.02)$, while changes in fat mass showed no correlation. 
a)

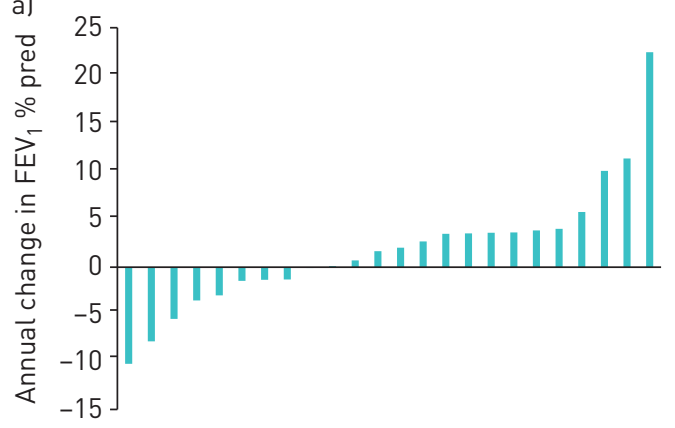

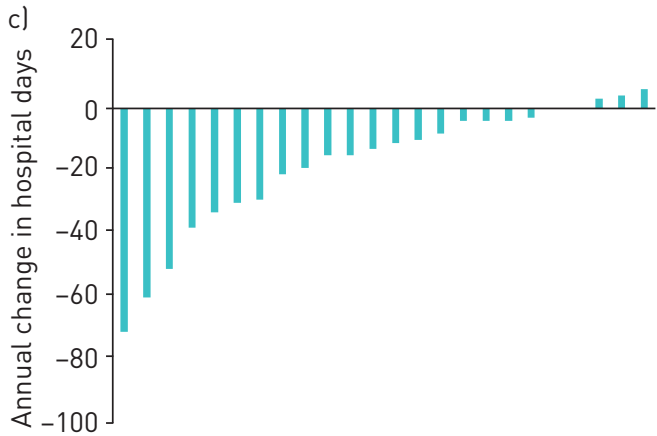

b)

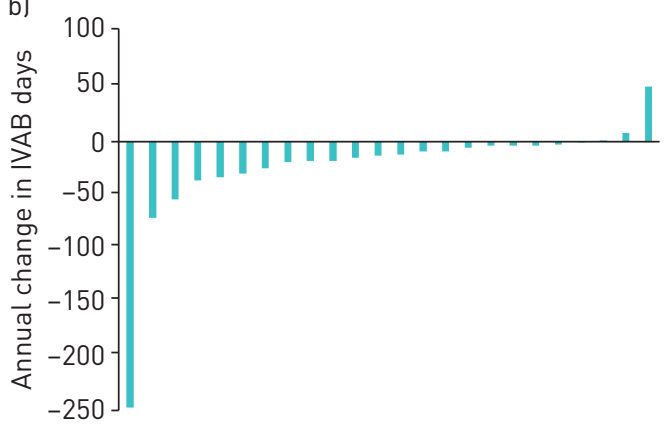

FIGURE 1 Waterfall plots. a) Plot for the slope of $\mathrm{FEV}_{1} \%$ pred in the year following starting LUM/IVA. Data show the individual patient $(n=24)$ changes in absolute $F E V_{1} \%$ pred in the year following LUM/IVA commencement, determined by linear regression of all pulmonary function from commencement to 1 year post. Mean change (slope \pm SE): $1.45 \%$ per year. b) Plot for changes in number of days of IVAB in the year following commencement of LUM/IVA compared with the year prior. Data show individual patient ( $\mathrm{n}=24$ ) changes in IVAB days (post-days-pre-days). Median (IQR) reduction: 13 (4-30) days. c) Plot for changes in number of days in hospital in the year following commencement of LUM/IVA compared with the year prior. Data show individual patient $(\mathrm{n}=24)$ changes in hospital days (post-days-pre-days). Median (IQR) reduction: 12 (3-30) days. $\mathrm{FEV}_{1}$ : forced expiratory volume in $1 \mathrm{~s}$; IVAB: intravenous antibiotic; LUM/IVA: lumacaftor/ivacaftor; IQR: interquartile range.

\begin{tabular}{|c|c|c|c|c|c|}
\hline & Baseline & 1 month & 6 months & 1 year & p-value \\
\hline Weight kg & $58.4 \pm 12.1$ & $59.0 \pm 11.3$ & $60.6 \pm 10.9 * *$ & $60.8 \pm 11.1$ & 0.0007 \\
\hline Fat-free mass $\mathbf{k g}$ & $46.1 \pm 11.2$ & $45.8 \pm 10.9$ & $46.7 \pm 11.0$ & $46.2 \pm 10.7$ & 0.49 \\
\hline Fat mass kg & $12.5 \pm 6.7$ & $13.5 \pm 6.4$ & $14.0 \pm 5.8^{* *}$ & $14.7 \pm 6.3^{* * *}$ & $<0.0001$ \\
\hline BMI $\mathrm{kg} \cdot \mathrm{m}^{-2}$ & $20.3 \pm 2.7$ & $20.5 \pm 2.4$ & $21.1 \pm 2.1^{*}$ & $21.2 \pm 2.3^{* *}$ & 0.0003 \\
\hline FFMI $\mathrm{kg} \cdot \mathrm{m}^{-2}$ & $15.9 \pm 2.5$ & $15.8 \pm 2.4$ & $16.1 \pm 2.4$ & $16.0 \pm 2.3$ & 0.48 \\
\hline $\mathrm{FMI} \mathrm{kg} \cdot \mathrm{m}^{-2}$ & $4.4 \pm 2.4$ & $4.8 \pm 2.4$ & $4.9 \pm 2.2^{* *}$ & $5.2 \pm 2.4^{* * *}$ & $<0.0001$ \\
\hline TBW L & $33.7 \pm 8.1$ & $33.6 \pm 7.7$ & $34.2 \pm 7.9$ & $33.9 \pm 7.8$ & 0.46 \\
\hline ICW L & $19.2 \pm 5.5$ & $19.2 \pm 5.5$ & $19.7 \pm 5.3$ & $19.5 \pm 5.2$ & 0.27 \\
\hline ECW L & $14.5 \pm 2.8$ & $14.4 \pm 2.7$ & $14.5 \pm 2.8$ & $14.4 \pm 2.8$ & 0.84 \\
\hline ECW \% TBW & $43.6 \pm 3.9$ & $43.3 \pm 3.4$ & $42.8 \pm 3.8$ & $43.0 \pm 3.5$ & 0.29 \\
\hline Phase angle ${ }^{\circ}$ & $4.8 \pm 0.9$ & $4.8 \pm 0.9$ & $4.7 \pm 1.4$ & $4.8 \pm 0.9$ & 0.08 \\
\hline
\end{tabular}

Data are presented as mean \pm SD, unless otherwise stated. BMI: body mass index; FFMI: fat-free mass index; FMI: fat mass index; TBW: total body water; ICW: intracellular water; ECW: extracellular water. \# : $\mathrm{n}=24$ for weight and BMI for all time-points, and for all data at 1 year; $n=23$ for all other measurements at baseline, 1 month and 6 month time-points (for one patient, useable bioelectrical impedance analysis could not be obtained at baseline and 1 month, and one patient missed the 6-month measurement). Individual $p$-values for change between baseline and that time-point (derived from the regression model with Bonferroni correction): *: $p<0.05 ;{ }^{* *}: p<0.005 ;{ }^{* * *}: p<0.0001$. Overall $p$-value is for the linear mixed effects regression model for effect of time on variables. 

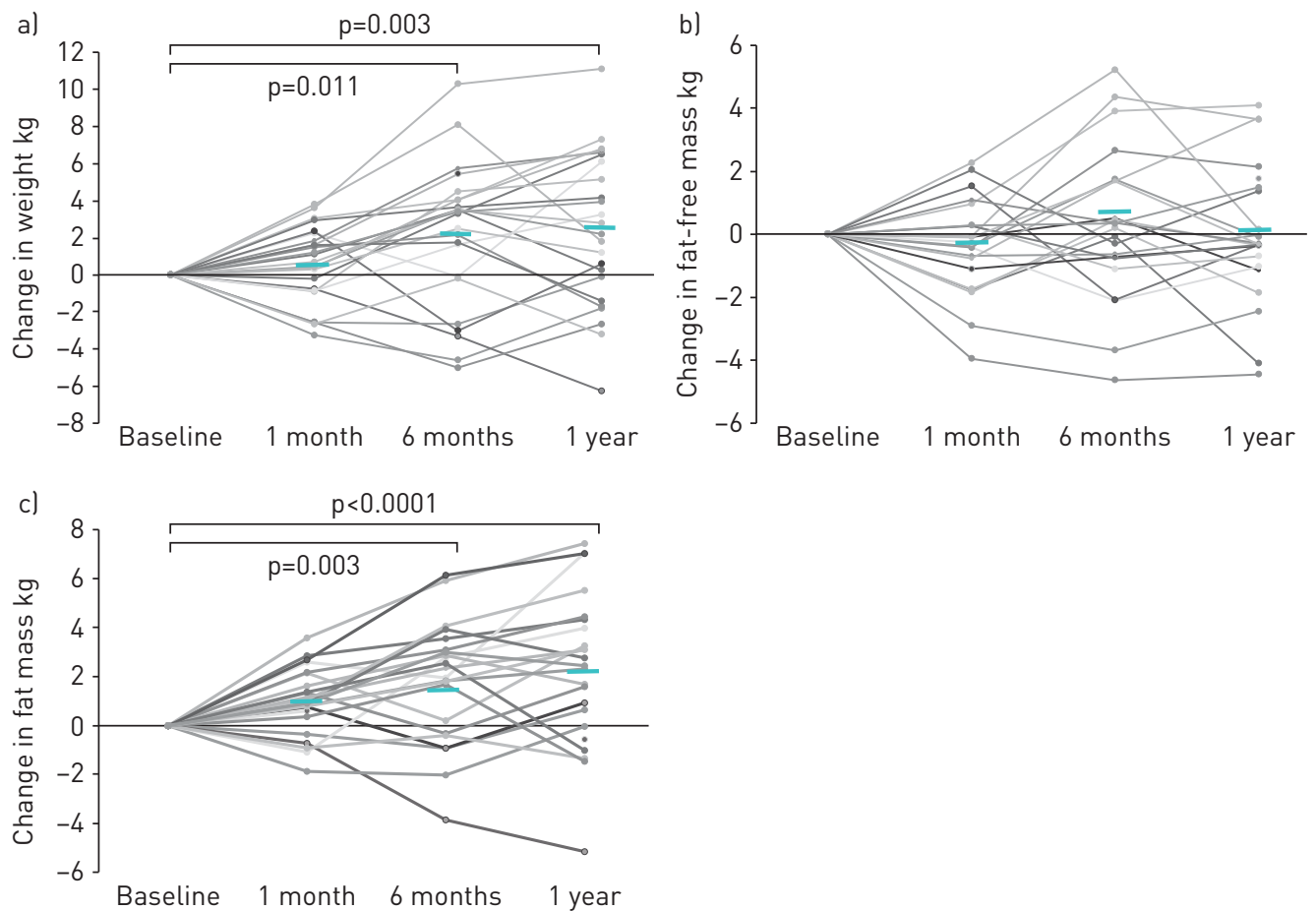

FIGURE 2 Body composition changes in 24 adults receiving lumacaftor/ivacaftor for 1 year: a) weight, b) fat-free mass and c) fat mass. Data show each individual measurement. Bars show mean change from baseline at each time-point. $p$-values indicate differences in mean values between time-points using linear mixed effects modelling with Bonferroni correction for multiple comparisons.

The reduction in hospitalisation days and IVAB usage between the years prior to and post-LUM/IVA were strongly correlated $(\rho=0.72, p=0.0001)$ in our patient cohort. However, no significant correlations were seen between changes in IVAB usage and changes in the slopes for pulmonary function parameters, nor for age or sex, indicating no clearly detectable clustering in the health status improvements following commencement of LUM/IVA (figure 3). Similar patterns were observed for changes in hospitalisation days (data not shown).

\section{Discussion}

In the present study we describe our real-world clinical experience with LUM/IVA treatment in a cohort of CF adults homozygous for the Phe508del mutation with severe lung disease. Although 31 adults were still on compassionate use LUM/IVA at the time of study assessment, only 24 had reached 1 year of LUM/IVA treatment and therefore were the focus of this report. Of the remaining nine patients who ceased LUM/IVA treatment, six (15\%) had ceased because of adverse events/intolerance (airway reactivity or significant deterioration in liver function tests), whereas three (7.5\%) ceased due to undergoing lung transplantation. The discontinuation rates for LUM/IVA in our cohort were therefore similar to, if not lower than, those quoted in the literature for patients with severe lung disease $[5,6,24-26]$. While the reasons for this cannot be conclusively identified, we believe that the model of care we established, including initiating LUM/IVA in the inpatient setting and regular outpatient review, maximised clinical stability, facilitated early identification and pre-emptive management of adverse effects, and supported adherence to LUM/IVA.

In our cohort of 24 patients who received LUM/IVA treatment for 1 year we report health stabilisation with significant improvements in CF-related clinical measures (frequency and severity of exacerbations in pulmonary sepsis requiring hospitalisation and IVAB treatment, and relative stabilisation in pulmonary function decline), significant improvements in weight and fat mass, and a reduction in the prevalence of severe underweight. These results both confirm and extend the findings from previous studies involving patients mild-to-moderate lung disease $[1,2,4,26]$ as well as those with severe lung disease $[5,6,25]$. Moreover, the overall reduction in pulmonary exacerbation rate post-LUM/IVA in our cohort of severe lung disease patients $\left(\mathrm{FEV}_{1} \%\right.$ pred $\left.<40 \%\right)$ and the improvement in average rate of decline in $\mathrm{FEV}_{1} \%$ pred at an individual level were not dissimilar to those found in larger studies of patients with $\operatorname{FEV}_{1} \%$ pred $>40 \%[1,2]$.

However, at the individual patient level, there was no clear clustering of improvement across clinically relevant domains. Hence, patients with the greatest reduction in health service utilisation were not 


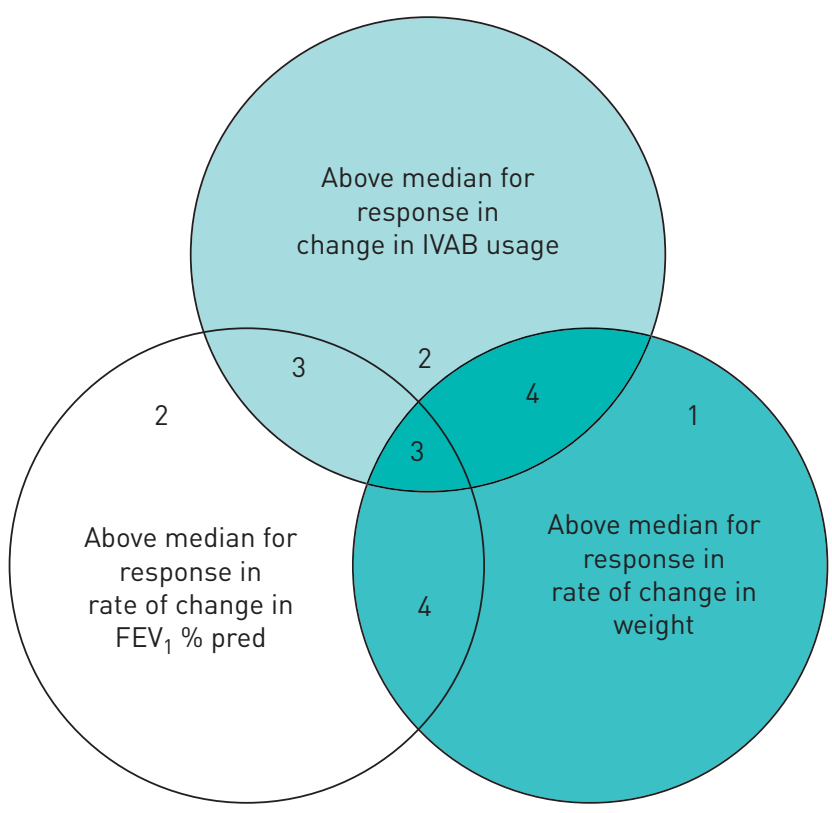

FIGURE 3 Venn diagram showing lack of clustering of clinical response domains for changes on lumacaftor/

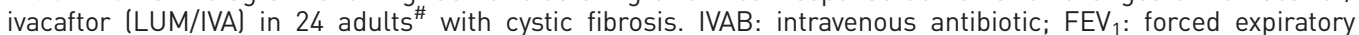
volume in $1 \mathrm{~s}$. Data have taken the median response for each domain (annual change in IVAB usage (days), rate of change in $\mathrm{FEV}_{1} \%$ pred and rate of change in weight $(\mathrm{kg})$ ). Numbers in the circles show patients with a response greater than the median change (where "response" is the change in rate between the year prior to starting LUM/IVA and the year following commencement). " : five patients had a response below the median change for all three domains.

consistently those with the greatest stabilisation in $\mathrm{FEV}_{1} \%$ pred decline nor were they necessarily associated with the greatest improvements in weight. These findings of improvements in various clinical domains as well patient-focused and health service-related outcomes underscore the importance of including these measures in both clinical trials and in the post-trial clinical evaluation setting where possible. Given the progressive rollout of CFTR modulator therapies including LUM/IVA, tezacaftor/ ivacaftor and elexacaftor/tezacaftor/ivacaftor, it can be argued that this will need to be matched with a parallel real-world evaluation. This will best inform the continuous evolution of clinical models of care for adults with $\mathrm{CF}$, especially as care shifts away from inpatient care and acute exacerbations to requiring a higher proportion of their hospital visits in the outpatient setting. Given that patients with $\mathrm{FEV}_{1}$ \% pred $<40 \%$ were also excluded from clinical trials of triple combination therapy for CF patients, real-world evaluation of its clinical effects in severe lung disease is also critical. Our data reporting on body composition changes on LUM/IVA will also provide comparative data for interpretation of the greater weight gain seen with triple combination therapy [27,28], which is particularly relevant given that excess adiposity is associated with normal-weight obesity [14].

Although our data indicated an expected positive correlation between the reduction in hospitalisation days and IVAB usage, changes in health service utilisation and pulmonary function were not correlated nor were improvements in nutritional parameters and pulmonary function status. The latter two findings suggest that the improvement in physiological measures following commencement of LUM/IVA is relatively discordant in severe lung disease. This may have significant mechanistic implications. Specifically, pathobiological drivers for exacerbations in airway infection, ongoing inflammation and catabolic processes at the very least are not completely overlapping and appear to be variably responsive to CFTR modulator intervention at the individual patient level. A recent biomarker study suggested heterogeneity in the response to CFTR modulator treatment [29]. As "real-world" experience with CFTR modulators accumulates, emerging information on variability in clinical responses will help guide clinical decision making.

The reversal of previous weight loss and stability of fat-free mass signals in our CF cohort with more severe lung disease treated with compassionate use LUM/IVA for 1 year is an important finding. Given that malnutrition is associated with poor outcomes both pre- and post-lung transplantation, interventions to maintain and improve nutritional status are key components of the management of patients with severe lung disease [30]. Our findings suggest that fat-free mass change was associated with reduced requirement for hospitalisation and parenteral antibiotics, although not necessarily associated with improvements in rates of pulmonary function decline. Although our real-world evaluation study was not designed to 
examine the mechanisms underpinning our observations and therefore cannot delineate the potential contribution of improvements in the anorectic/catabolic state to improvements in nutritional parameters, it does nevertheless suggest that these and other factors are likely to be important. These factors may include improvements in gastrointestinal absorption of nutrients, increased nutrient intake, improvements in neurohormonal stress metabolism, reduction in resting energy expenditure, and improvements in intracellular energetics and metabolic efficiencies, with most of the evidence in support of mechanisms underlying nutritional improvements coming from studies on ivacaftor [31-33]. There was relatively minimal change seen in ECW as a percentage of TBW across the cohort throughout the 1-year period, whereas the weight and fat mass gains generally plateaued between 6 months and 1 year.

A major strength of this study is the real-world evaluative aspects of compassionate use LUM/IVA in a cohort of severe lung disease patients followed for 1 year in a large, single adult CF centre. By its very nature, this study's clear trade-off is that it was not prospectively designed, randomised and fully protocol driven, and it was not feasible to have a control (no treatment) group. Thus, we cannot rule out that for this group of patients with severe lung disease the hope and opportunity offered by access to LUM/IVA may have contributed to improvements in motivation of adherence to usual treatment regimens. However, the relatively tight regulation associated with gaining access to compassionate use LUM/IVA and monitoring its use as well as the single-centre approach significantly helped reduce, but not eliminate, the possibility of healthcare decision-related biases. Furthermore, a single-centre study allowed inclusion of body composition data for sequential monitoring, available at our centre, but not routinely in all CF centres. With this in mind, our report included complete follow-up and data acquisition for all patients except for only three out of 96 body composition data points missing. Additionally, the potential for biasing pulmonary function testing by having more measurements in the sicker patients was statistically accounted for in our analysis, thereby minimising any potential impact on this result.

Another strength is inclusion of body composition monitoring, which is infrequently reported in clinical trials. Therefore, our data extend knowledge beyond the predominantly weight-based nutritional outcomes for CFTR modulator therapies available to date. The lack of further systematic assessment of nutritional and energy parameters as well as sweat chloride testing throughout the study period is a limitation to our final analysis. Whereas the use of bioelectrical impedance analysis proved to be practical and feasible (no recurrent cost, no exposure to radiation and available on demand at our centre) in our study and has high precision, it was not feasible to perform more detailed metabolic testing such as indirect calorimetry or sweat chloride testing nor obtain repeated food records for dietary intake analysis [32] in a busy clinical setting. The impact of CFTR modulators on quality of life, physical functioning and social participation are important patient-centred outcomes, but were outside the scope of the current report; therefore, we are unable to comment on any correlations between clinical or nutritional findings and these parameters.

In conclusion, our real-world evaluation study confirmed the feasibility and benefits of LUM/IVA in a significant proportion of CF patients with severe lung disease. Moreover, these benefits occurred over several clinical domains (infective exacerbations requiring hospitalisation for parenteral antibiotic treatment, rates of pulmonary function decline and nutritional parameters) that were not necessarily clustered in individual CF patients. Whether these findings will be similar with the newer CFTR modulator therapies applicable to this cohort of patients will be of major interest, as will the question of whether patients who did not tolerate LUM/IVA will be able to tolerate and achieve clinical benefit from these newer agents. To this end, our data support a case for completing real-world evaluations of these new medications to maximise understanding and insights that may be associated with their prolonged use. Such evaluations at the very least should include a systematic clinical framework within which a range of investigative assessments that have a relatively high index of capturing important signals can be readily performed in an outpatient setting at an acceptable cost.

Acknowledgements: The authors acknowledge the contribution of Bhey Fox, Sarah Ryan (Nutrition Dept, Alfred Hospital, Melbourne, Australia) and Denise Clark (Cystic Fibrosis Service, Alfred Hospital, Melbourne, Australia) to acquiring body composition data.

Conflict of interest: S.J. King reports a lecture fee from Vertex Pharmaceuticals in November 2017, outside the submitted work. D. Keating reports consultation fees from Vertex Pharmaceuticals, outside the submitted work. E. Williams reports a consultancy fee from Vertex Pharmaceuticals for a paid, 1-day workshop, outside the submitted work. E. Paul has nothing to disclose. B.M. Borg has nothing to disclose. F. Finlayson has nothing to disclose B.M. Button reports lecture and consultancy fees from Vertex Pharmaceuticals, outside the submitted work. J.W. Wilson reports consultancy and lecture fees from Vertex Pharmaceuticals, outside the submitted work. T. Kotsimbos reports lecture fees from Vertex Pharmaceuticals, outside the submitted work.

\section{References}

1 Wainwright CE, Elborn JS, Ramsey BW. Lumacaftor-ivacaftor in patients with cystic fibrosis homozygous for Phe508del CFTR. N Engl J Med 2015; 373: 1783-1784. 

patients with cystic fibrosis homozygous for Phe508del CFTR by pulmonary function subgroup: a pooled analysis. Lancet Respir Med 2016; 4: 617-626.

3 Southern KW, Patel S, Sinha IP, et al. Correctors (specific therapies for class II CFTR mutations) for cystic fibrosis. Cochrane Database Syst Rev 2018; 8: CD010966.

4 Konstan MW, McKone EF, Moss RB, et al. Assessment of safety and efficacy of long-term treatment with combination lumacaftor and ivacaftor therapy in patients with cystic fibrosis homozygous for the F508del-CFTR mutation (PROGRESS): a phase 3, extension study. Lancet Respir Med 2017; 5: 107-118.

5 Murer C, Huber LC, Kurowski T, et al. First experience in Switzerland in Phe508del homozygous cystic fibrosis patients with end-stage pulmonary disease enrolled in a lumacaftor-ivacaftor therapy trial - preliminary results. Swiss Med Wkly 2018; 148: w14593.

6 Taylor-Cousar JL, Jain M, Barto TL, et al. Lumacaftor/ivacaftor in patients with cystic fibrosis and advanced lung disease homozygous for F508del-CFTR. J Cyst Fibros 2018; 17: 228-235.

7 Horsley A, Barry P. Orkambi in patients with severe disease - bumps in the road to CFTR modulation. $J$ Cyst Fibros 2017; 16: 311-312.

8 Ramos KJ, Smith PJ, McKone EF, et al. Lung transplant referral for individuals with cystic fibrosis: Cystic Fibrosis Foundation consensus guidelines. J Cyst Fibros 2019; 18: 321-333.

9 Cystic Fibrosis Foundation. Patient Registry 2018 Annual Data Report. Bethesda, CFF, 2019.

10 Calella P, Valerio G, Thomas M, et al. Association between body composition and pulmonary function in children and young people with cystic fibrosis. Nutrition 2018; 48: 73-76.

11 King SJ, Nyulasi IB, Strauss BJG, et al. Fat-free mass depletion in cystic fibrosis: associated with lung disease severity but poorly detected by body mass index. Nutrition 2010; 26: 753-759.

12 Steinkamp G, Wiedemann B. Relationship between nutritional status and lung function in cystic fibrosis: cross sectional and longitudinal analyses from the German CF quality assurance (CFQA) project. Thorax 2002; 57: 596-601

13 Sheikh S, Zemel BS, Stallings VA, et al. Body composition and pulmonary function in cystic fibrosis. Front Pediatr 2014; 2: 33.

14 Alvarez JA, Ziegler TR, Millson EC, et al. Body composition and lung function in cystic fibrosis and their association with adiposity and normal-weight obesity. Nutrition 2016; 32: 447-452.

15 Bolton CE, Ionescu AA, Evans WD, et al. Altered tissue distribution in adults with cystic fibrosis. Thorax 2003; 58: 885-889.

16 Ionescu AA, Evans WD, Pettit RJ, et al. Hidden depletion of fat-free mass and bone mineral density in adults with cystic fibrosis. Chest 2003; 124: 2220-2228.

17 Alicandro G, Bisogno A, Battezzati A, et al. Recurrent pulmonary exacerbations are associated with low fat free mass and low bone mineral density in young adults with cystic fibrosis. J Cyst Fibros 2014; 13: 328-334.

18 Ionescu AA, Nixon LS, Luzio S, et al. Pulmonary function, body composition, and protein catabolism in adults with cystic fibrosis. Am J Respir Crit Care Med 2002; 165: 495-500.

19 King SJ, Nyulasi IB, Bailey M, et al. Loss of fat-free mass over four years in adult cystic fibrosis is associated with high serum interleukin-6 levels but not tumour necrosis factor-alpha. Clin Nutr 2014; 33: 150-155.

20 Sermet-Gaudelus I, Souberbielle JC, Azhar I, et al. Insulin-like growth factor I correlates with lean body mass in cystic fibrosis patients. Arch Dis Child 2003; 88: 956-961.

21 White H, Morton A, Peckham D. Impact of enteral tube feeding on body fat and lean body mass. J Cyst Fibros 2014; 13: Suppl., S101.

22 Bosy-Westphal A, Schautz B, Later W, et al. What makes a BIA equation unique? Validity of eight-electrode multifrequency BIA to estimate body composition in a healthy adult population. Eur J Clin Nutr 2013; 67: Suppl $1, \mathrm{~S} 14-\mathrm{S} 21$.

23 Cederholm T, Bosaeus I, Barazzoni R, et al. Diagnostic criteria for malnutrition - an ESPEN Consensus Statement. Clin Nutr 2015; 34: 335-340.

24 Hubert D, Chiron R, Camara B, et al. Real-life initiation of lumacaftor/ivacaftor combination in adults with cystic fibrosis homozygous for the Phe508del CFTR mutation and severe lung disease. J Cyst Fibros 2017; 16: 388-391.

25 Tong K, Barker D, France M, et al. Lumacaftor/ivacaftor reduces exacerbations in adults homozygous for Phe508del mutation with severe lung disease. J Cyst Fibros 2020; 19: 415-420.

26 Burgel PR, Munck A, Durieu I, et al. Real-life safety and effectiveness of lumacaftor-ivacaftor in patients with cystic fibrosis. Am J Respir Crit Care Med 2020; 201: 188-197.

27 Heijerman HGM, McKone EF, Downey DG, et al. Efficacy and safety of the elexacaftor plus tezacaftor plus ivacaftor combination regimen in people with cystic fibrosis homozygous for the F508del mutation: a double-blind, randomised, phase 3 trial. Lancet 2019; 394: 1940-1948.

28 Middleton PG, Mall MA, Drevinek P, et al. Elexacaftor-tezacaftor-ivacaftor for cystic fibrosis with a single Phe508del allele. N Engl J Med 2019; 381: 1809-1819.

29 Masson A, Schneider-Futschik EK, Baatallah N, et al. Predictive factors for lumacaftor/ivacaftor clinical response. J Cyst Fibros 2019; 18: 368-374.

30 Kapnadak SG, Dimango E, Hadjiliadis D, et al. Cystic Fibrosis Foundation consensus guidelines for the care of individuals with advanced cystic fibrosis lung disease. J Cyst Fibros 2020; 19: 344-354

31 Houwen RHJ, van der Woerd WL, Slae M, et al. Effects of new and emerging therapies on gastrointestinal outcomes in cystic fibrosis. Curr Opin Pulm Med 2017; 23: 551-555.

32 Sainath NN, Schall J, Bertolaso C, et al. Italian and North American dietary intake after ivacaftor treatment for cystic fibrosis gating mutations. J Cyst Fibros 2019; 18: 135-143.

33 Stallings VA, Sainath N, Oberle M, et al. Energy balance and mechanisms of weight gain with ivacaftor treatment of cystic fibrosis gating mutations. J Pediatr 2018; 201: 229-237. 